\title{
Cases of Inversion of Uterus.
}

\author{
By R. Drummond Maxwell, M.D.
}

CASEs of inversion of uterus warrant a clinical record for several reasons :-

(I) The extraordinary rarity. No cases have been admitted to the London Hospital for the last fifteen years.

(2) The divergent views expressed on the treatment of such cases.

(3) A tendency in modern literature to invoke surgical treatment as opposed to the classical treatment by reposition associated with the names of Aveling and Matthew Duncan.

CASE I. The first case referred to is of the " chronic" variety, and concerns a patient, aged 24, married two years, primigravida; seen by Dr. Lewers, July igi2. No special history was obtained from the patient. Instrumentally delivered under chloroform. The patient had no knowledge of third stage complications.

She was admitted owing to free hæmorrhage three days after labour. A mass the size of a foetal head projected through the cervix into the vagina. There was no obvious sepsis; the uterine surface was clean; there was retention of urine, $35 \mathrm{oz}$. were drawn off by the catheter. The peritoneal "cup " of the inverted uterus was easily felt. Dr. Lewers saw the patient next day and tried manual reposition, but did not persist in this owing to severe pain caused thereby.

The hæmorrhage was checked in hospital during the next three or four days by repeated local douches. She was sent to a convalescent home fourteen days later, to come up again in a few weeks for further treatment. She did not, however, turn up until January 1913 (that is, four months' interval instead of one month), stating that she was fairly well until her period came on at the fourth month, and then she bled so freely that she became alarmed, and returned to hospital. On examination the involution was found to be fairly good, although the fundus was still as large as a golf ball, and projected for $2 \frac{1}{2}$ in. into the vagina. Under chloroform Aveling's repositor was applied, with a large cup which fitted easily over the protruding fundus. It was left in overnight and taken oit next morning. A considerable reduction of the inversion was noted. In forty hours' time, after five reapplications of the repositor, the fundus was now well within the cervical canal. To avoid difficulty in removal of the cup from the reinverted uterus a 
smaller cup was used for the final stages, and its ascent carefully watched. In forty-three and a half hours from the commencement of these manipulations the uterus was found to be in its normal position. An interesting rise of temperature commenced from the day of final reposition of uterus, and lasted for eight days, varying between $103^{\circ} \mathrm{F}$. and $99^{\circ} \mathrm{F}$. This temperature gradually subsided, and the last three weeks of the patient's stay in hospital was apyrexial.

The patient has recently been heard of, and is in good health. No subsequent pregnancy has been recorded.

CASE II. The second case is of the acute variety. The patient was admitted to hospital on October 21, 1914, with a very grave anæmia and serious flooding. She had been delivered three days before, and had been sent to hospital with the diagnosis of septicæmia. The patient's appearance and condition before vaginal examination were most suggestive of that diagnosis.

Details of the obstetric history are as follows: The patient, a primigravida, aged 24 , had been delivered three days previously. Forceps had been applied. There was some difficulty in connection with the third stage. The placenta had not apparently been removed manually.

I examined the patient at I p.m. The bladder was emptied by catheter. A large mass was felt in the vagina and delivered externally. It was smooth over the greater part of the surface, but its lower pole was covered with necrotic tissue, fibrin, and placental shreds, which were still firmly adherent. There was intense foetor, and very free hæmorrhage during examination. Per abdomen the peritoneal " cup " could be readily detected. It was impossible at first to get even a finger-tip from below through it. The inversion appeared to be "complete." From below, the uterine body, which was long and flask-shaped, narrowing at its neck to the calibre of a five-shilling piece, was firmly squeezed with hot towels to reduce its œdematous bulk. The foul clots and shreds were picked off the fundus, but it was difficult to determine the right plane of cleavage of the placental relics, and as this appeared to run rather too deep into the uterine wall further separation was not persisted in, and attempt at reduction was begun by pushing up a small portion at the side, and inverting it through the cup. One finger-tip carrying the lateral wall of the uterus in front of it was eventually felt per abdomen. This process was proceeded with all round the ring from below, till eventually four fingers pushed through the ring from below could easily be felt by the abdomen. The fundus of the uterus, which now lay about the level of the cervix, gave rise to little further difficulty. The complete reposition of the uterus from first to last took about forty minutes. A very considerable amount 
of force had to be used at the start to dilate the ring from below, but once this had been effected no further difficulty was experienced. There was little shock on the whole following these manipulations.

The subsequent progress of the case was as follows: The patient stood the manual reposition well on the whole considering her profoundly debilitated condition. The next day there was naturally very considerable hypogastric tenderness, for the counterpressure exerted from above had not been inconsiderable. Although the discharge from the uterus still remained reddened, there was at no time any further loss that could be considered severe. The discharge remained foul, though even this improved considerably with intra-uterine irrigation. Patient remained in a very weak exhausted state for the next seven days, and a diarrhœa, undoubtedly of toxic origin, retarded her convalescence. On the twelfth day the patient developed symptoms of a pulmonary embolism, sudden severe pain on the left side, crepitations, and rusty sputum. These signs were not progressive, and the patient still seemed to have a desperate chance of recovery till on the sixteenth day a sudden left hemiplegia occurred and she died in a few hours. A blood culture taken on the thirteenth day showed no positive growth of microorganisms. The hemiplegia, in the absence of a post-mortem investigation, must therefore be attributed either to a thrombosis secondary to her anæmia or to cerebral embolism.

Several points of interest arise for discussion. It will be noticed that both cases were accompanied by retention of urine. This is remarkable, considering the fact that the bladder practically never shares in the displacement, though its anatomical relations with the uterus would rather lead one to believe that this would always occur. On the whole, I am inclined to attribute the retention of urine, certainly in the acute case, to collapse and exhaustion.

The points I would submit for discussion on the treatment of the acute case are two in number :-

(I) Is it advisable as a general procedure to attempt reduction of an acutely inverted uterus as late as twenty-four hours after this displacement has occurred?

In answer to this question, I would suggest that in my opinion, as a general rule, such treatment will not be wise, but a complicating factor, such as excessive bleeding from the tumour, may always confuse a clear decision on this point (as happened in my case). I felt I could not, with an easy conscience, abandon to expectant treatment a patient who had already suffered grave bleeding-and bleeding, moreover, which was still persisting in a lesser degree.

(2) Is manual reposition of an inverted uterus (in the acute 
stage) indicated in the presence of septic metritic changes in its wall?

Were this the sole factor to be considered the answer would be, I think, quite definitely, that such manipulations are strongly contraindicated. But one of the unfortunate (or fortunate) considerations of so many urgent obstetric problems is that the accoucheur is left so much wiser after the event than before. Here was a uterus that appeared on the third day to be well-nigh strangulated. Its neck was narrowed down to the calibre of a five-shilling piece, and comprised in its circumference not only the cervical walls but the essential blood supply of the uterus in the broad ligaments. Perhaps I feared excessively a total necrosis of the lower pole of the uterus which would not have occurred, but in any case I am now convinced that, regarded from the point of view of septic uterine changes, the expectant attitude is the one that should have been adopted. That course has been followed out in a subsequent case admitted (under the care of my colleague, Dr. Russell Andrews) in the acute stage. Involution is, I believe, proceeding satisfactorily, and the patient will shortly be readmitted for reduction of the "chronic" displacement with Aveling's repositor.

It is a point of some interest to note that after an interval of close upon eighteen years three cases of the " acute" variety have been admitted in less than two years to the London Hospital. I believe on the whole that this displacement is not so rare as statistics would suggest, and that view is, I think, confirmed by the numbers of Fellows present to-night who have related their personal experience of the condition. 